\title{
4D-CT for Preoperative Localization of Abnormal Parathyroid Glands in Patients with Hyperparathyroidism: Accuracy and Ability to Stratify Patients by Unilateral versus Bilateral Disease in Surgery-Naive and Re-Exploration Patients
}

H.R. Kelly, L.M. Hamberg, and G.J. Hunter

\begin{abstract}
BACKGROUND AND PURPOSE: 4D-CT is an emerging technique that uses high-resolution images, multiplanar reformats, and perfusion characteristics to identify abnormal parathyroid glands in patients with hyperparathyroidism. This study evaluates the accuracy of 4D-CT for localization and lateralization of abnormal parathyroid glands in preoperative planning for minimally invasive parathyroidectomy vs bilateral neck exploration at a tertiary referral center.
\end{abstract}

MATERIALS AND METHODS: Radiology, pathology, and operative reports were retrospectively reviewed for 208 patients with hyperparathyroidism who underwent 4D-CT and parathyroid surgery between May 2008 and January 2012. 4D-CT performance in localizing side and site was determined by use of surgical and pathologic findings as a reference.

RESULTS: Of 208 patients, 155 underwent initial surgery and 53 underwent re-exploration parathyroid surgery. No lesions were found in 8 patients (3.8\%). A total of 284 lesions were found in 200 patients; 233 were correctly localized by 4D-CT ( $82.0 \%)$. Of the 200 patients with parathyroid lesions, 146 underwent unilateral and 54 bilateral neck exploration. 4D-CT correctly identified unilateral vs bilateral disease in 179 (89.5\%) of 200. 4D-CT correctly localized parathyroid lesions in 126 of the unilateral cases (86.3\%). In the re-exploration cohort, 4D-CT correctly identified unilateral vs bilateral disease in $46(95.8 \%)$ of 48 . There was no statistically significant difference in subgroups stratified by surgery type (primary or subsequent) and number of scan phases ( 3 or 4$)(P>.56)$.

CONCLUSIONS: 4D-CT leverages modern high-resolution CT scanning and dynamic contrast enhancement to localize abnormal parathyroid glands in patients with hyperparathyroidism of any cause and can be used for planning minimally invasive parathyroidectomy vs bilateral neck exploration.

ABBREVIATIONS: $E M R=$ electronic medical record; $\mathrm{PPV}=$ positive predictive value

$\mathrm{H}$ yperparathyroidism is caused by overproduction of parathyroid hormone by a single adenoma, multigland hyperplasia or multiple adenomata. In primary hyperparathyroidism, a single adenoma is the cause in most cases $(75 \%-85 \%)$, with multiple adenomas or multigland hyperplasia found in a smaller subset of patients (approximately $4 \%$ and $10 \%$, respectively). ${ }^{1,2}$ In secondary and tertiary hyperparathyroidism, excess parathyroid hor-

Received December 20, 2012; accepted after revision April 5, 2013.

From the Division of Diagnostic Neuroradiology (H.R.K., G.J.H.), Massachusetts General Hospital/Harvard Medical School, Boston, Massachusetts; Department of Radiology (H.R.K.), Massachusetts Eye and Ear Infirmary/Harvard Medical School, Boston, Massachusetts; and Department of Radiology (L.M.H.), Brigham and Women's Hospital/Harvard Medical School, Boston, Massachusetts.

Recipient of the Radiologist-In-Training Award for results previously presented in part at the Annual Meeting of the American Society of Head and Neck Radiology, September 2011; San Diego, California.

Please address correspondence to Hillary R. Kelly, MD, Massachusetts General Hospital, Division of Neuroradiology, 55 Fruit St, GRB-273A, Boston, MA 02114; e-mail: hkelly2@partners.org

http://dx.doi.org/10.3174/ajnr.A3615 mone is usually secreted by multiple hyperplastic glands. ${ }^{3}$ The only therapeutic option for complete cure of primary hyperparathyroidism is surgery, with reported success rates of greater than $95 \%$ for the reference standard of bilateral neck exploration performed by an experienced surgeon. ${ }^{4}$ Minimally invasive parathyroidectomy with a unilateral surgical approach is rapidly becoming the standard of care in an effort to lower complication rates, reduce costs, shorten hospital stays, and improve cosmetic results. ${ }^{4-6}$ Preoperative localization and lateralization of abnormal parathyroid glands are integral to the performance of unilateral minimally invasive parathyroidectomy. ${ }^{7}$ Preoperative imaging is also integral to operative planning in patients with recurrent hyperparathyroidism undergoing redo surgery. ${ }^{8}$ At most institutions, the current favored approach to preoperative imaging for hyperparathyroidism includes a combination of sonography and technetium Tc99m sestamibi scanning, with a reported combined sensitivity of $74 \%-95 \%$ for single-gland disease. ${ }^{8}$ However, the sensitivities reported for multigland disease and multiple adeno- 
mata are much lower, in the range of $15 \%-35 \%$ for sonography and 30\%-44\% for technetium Tc99m sestamibi. ${ }^{8}$

$4 \mathrm{D}-\mathrm{CT}$ has been proposed as an alternative or adjunctive method for preoperative localization of abnormal parathyroid glands in patients with primary hyperparathyroidism. ${ }^{9-13}$ Multiple recent studies have indicated that $4 \mathrm{D}-\mathrm{CT}$ may be especially useful for preoperative localization in patients with negative or inconclusive results on sonography and technetium Tc99m sestamibi studies, reoperative parathyroid patients, patients with mild hypercalcemia, and in patients with multigland disease. ${ }^{14-18}$ However, previously published reports have predominantly evaluated cohorts with small numbers of patients and have focused on specific patient populations with strict exclusion criteria. One study with a large group of patients included only those with sporadic primary hyperparathyroidism who had a single adenoma identified by 4D-CT and were undergoing initial parathyroidectomy, excluding patients with multigland disease, prior surgery, concomitant thyroid disease, mediastinal ectopic parathyroid glands, and multiple endocrine neoplasia syndromes. ${ }^{12}$

The purpose of our study was to assess the accuracy of 4D-CT for localizing and characterizing the number of parathyroid adenomas and/or hyperplasia in a large group of unselected patients evaluated at a tertiary care center. Our hypothesis was that 4D-CT may be used to accurately predict the site and number of parathyroid lesions and thus allow planning for a minimally invasive, unilateral surgical approach vs a conventional bilateral neck exploration. Furthermore, we hypothesized that the accuracy of $4 \mathrm{D}-\mathrm{CT}$ is not reduced in those patients who have undergone prior parathyroid surgery.

\section{MATERIALS AND METHODS Patients}

This retrospective study was approved by our institutional review board, waiving consent in accordance with the Health Insurance Portability and Accountability Act. We began performing 4D-CT examinations at our institution in May 2008. Patients were included in the study if they carried a clinical and biochemical diagnosis of hyperparathyroidism, had 4D-CT for preoperative localization between May 2008 and July 2011, and subsequently underwent surgical exploration between May 2008 and January 2012. Inclusion criteria also included the availability of the operative report and final pathology report. Patient demographic data including age and sex were obtained from the electronic medical record (EMR).

\section{CT Technique}

Examinations were performed on a 16- or 64-multidetector row CT scanner (LightSpeed 16 Pro and Discovery CT750 HD; GE Healthcare, Milwaukee, Wisconsin) with use of the following standardized protocol. Patients were supine, head first in the scanner. The scanner manufacturer-supplied head holder was used for all scans. Patients' arms were extended caudally by use of manufacturer-supplied straps, specifically designed to reduce shoulder artifacts. Scanning extended from the carina to the maxillary teeth. Scanning parameters for each phase were $140 \mathrm{kVp}$, 180-300 mA by automatic exposure control (Auto-mA algorithm; GE Healthcare), 1-second rotation time, 1.375 pitch, a $0.625-\mathrm{mm}$ detector configuration with a beam width of $10-\mathrm{mm}$ for the 16-section scanner and $40 \mathrm{~mm}$ for the 64 -section scanner, and $1.25-\mathrm{mm}$ section thickness reconstructed at $1-\mathrm{mm}$ centers. The first phase was acquired before the administration of intravenous contrast material. Iodinated contrast material (Isovue 370; Bracco Diagnostics, Princeton, New Jersey) was then administered at a dose of $100 \mathrm{~mL}$ at an infusion rate of $4 \mathrm{~mL}$ per second via an 18-gauge catheter, followed by $40 \mathrm{~mL}$ of saline at an infusion rate of $4 \mathrm{~mL}$ per second. The second phase was acquired $30 \mathrm{sec}-$ onds after the start of intravenous contrast administration. A third delayed phase was acquired 30 seconds after the completion of the second phase (approximately 45-48 seconds after the start of the injection of contrast, depending on the length of the patient, table speed, and gantry rotation). For the examinations performed between May 2008 and June 9, 2010, a fourth "late-delayed" phase was acquired 45 seconds after the completion of the third "early delayed" phase. Only the initial 3 phases were acquired for all examinations performed between June 10, 2010, and June 2011.

\section{Image Processing and Analysis}

Standardized postprocessing was performed on all studies yielding multiplanar reformations, including a "true axial" plane parallel to the vocal cords, coronal, and sagittal planes orthogonal to the true axial plane, as well as bilateral anterior sagittal oblique planes parallel to the sternocleidomastoid muscles. All examinations and images were reviewed prospectively on a PACS workstation (Impax 5.3; Agfa HealthCare, Greenville, South Carolina) by a board-certified neuroradiologist (G.J.H.) with more than 17 years of experience and a Certificate of Added Qualification in neuroradiology. Additional 3D postprocessing, kinetic analysis, and volume-rendered images were also created and were reviewed as deemed necessary for image analysis by the interpreting neuroradiologist and/or as requested by the referring surgeon (Advantage Windows Workstation, ADW 4.2; GE Healthcare). A formal report was entered into the EMR for each study, with a detailed anatomic description of the location of the abnormal parathyroid lesion(s), if any were identified. Biochemical information, clinical history, and prior sonography and technetium Tc99m sestamibi imaging results (if available) were reviewed at the time of image interpretation.

\section{Retrospective Review of Imaging Results}

For each patient who met the criteria for inclusion into our study, the radiology report was retrieved from the EMR, and the side and anatomic location of the abnormal parathyroid lesion(s) were recorded by a neuroradiologist (H.R.K.) without knowledge of the operative findings. Localization of presumptive parathyroid lesions was with respect to the midline as defined by the trachea and was extended vertically into the mediastinum. Anatomic localization was refined by reference to the adjacent thyroid gland and the sternal notch or level of the clavicular heads. These landmarks are used by the surgeons to plan the correct access for adenoma resection. If the patient had prior surgery, any clips marking resected tissue were also used as reference points. When necessary and appropriate, additional reference points included the level of the carotid bifurcation, the hyoid bone, or mediastinal structures 
such as the aortic arch and carina. No reinterpretation of the images was performed at the time of this retrospective review. The results of any prior sonography and technetium Tc99m sestamibi imaging were also retrieved from the EMR, and the side and anatomic location of the parathyroid lesion(s) were recorded as indicated in the official reports. In a typical setting, the anatomic location provided by sonography or technetium Tc99m sestamibi imaging was with respect to the thyroid gland.

\section{Retrospective Review of Operative and Pathologic Results}

The surgical findings, as recorded in the operative note in the EMR, were used as the reference standard for final anatomic location of parathyroid lesions. Surgical nomenclature for the location of abnormal tissue was with reference to the thyroid gland or, if the lesion was ectopic, with reference to mediastinal or hyoid structures as appropriate, and matched the classification used by the radiologists. The pathologic findings were used as the reference standard for a definitive diagnosis of abnormal parathyroid tissue. The operative and pathology reports were retrieved from the EMR, and the findings were recorded for each patient in a separate data base blinded to the imaging findings. The anatomic location, as labeled by the surgeon when submitted to pathology, was also recorded. If multiple lesions were identified and removed, all lesions were recorded. This data base was then merged with the imaging results. The $4 \mathrm{D}-\mathrm{CT}$, sonography, and/or technetium Tc99m sestamibi studies were recorded as concordant with the operative and pathologic results if a parathyroid lesion was removed from the same location (relative to anatomic landmarks) as predicted by that imaging study.

\section{Statistical Analysis}

Excel 2011 (Microsoft Corporation, Redmond, Washington) was used for all statistical analysis. Accuracy and positive predictive value (PPV) of localization were determined as the number of patients in whom each imaging technique correctly identified the anatomic location of the pathology-proven lesion(s), expressed as a percentage of the total number of abnormal parathyroid glands found and removed at surgery. In patients with multiple lesions, correct identification of the location of each lesion by the imaging study was required for it to be considered concordant with surgery and pathology. Patients with mediastinal lesions were included in the localization analysis. To evaluate any potential effect on the results because of the change in protocol from 4-phase to 3 -phase, we repeated localization analyses with the patient cohort stratified by the 2 protocol subgroups. In these 2 subgroups, we undertook further stratification to distinguish between patients undergoing parathyroid surgery for the first time and those who had undergone prior parathyroid surgery. The Fisher exact test was used to test the null hypothesis of no difference between subgroups. A $P$ value of $<.05$ was considered significant.

\section{RESULTS}

A total of 275 patients underwent $4 \mathrm{D}$-CT scans in the study period. Of these, 67 did not proceed to surgery and were excluded from further evaluation. The remaining 208 patients all had radiologic, operative, and pathology reports available in the EMR and were analyzed. There were 30 men (mean age, 57.3 years; median age, 57.5 years; age range, $21-82$ years) and 178 women (mean age, 59.8 years; median age, 61 years; age range, 18-87 years).

There were 155 patients who underwent primary surgery. Of these, 90 had a 4-phase 4D-CT scan, and 65 patients underwent a 3 -phase $4 \mathrm{D}$-CT scan. In the 4-phase subgroup, 140 abnormal parathyroid glands were found at surgery in 89 patients, and 109 lesions were correctly localized with an accuracy of 77.9\% (109/ 140). 4D-CT identified 18 potential lesions that were not adenomatous or hyperplastic, giving a PPV of $85.8 \%$ (109/127). In the 3-phase subgroup, 93 parathyroid lesions were found at surgery in 63 patients. A total of 81 lesions were correctly localized with an accuracy of $87.1 \%$ (81/93). 4D-CT identified 10 potential lesions that were not adenomas, giving a PPV of $89.0 \%$ (81/91). No significant difference was observed between these 2 populations $(P>.57)$.

There were 53 patients who underwent a second surgery. Of these, 36 patients had a 4-phase 4D-CT scan and 17 patients underwent a 3-phase 4D-CT scan. In the 4-phase subgroup, 32 parathyroid lesions were found at surgery in 33 patients, and 27 lesions were correctly localized with an accuracy of $84.4 \%$ (27/32). 4D-CT identified 10 potential lesions that were not adenomas, giving a PPV of $73.0 \%$ (27/37). In the 3-phase subgroup, 19 parathyroid adenomas were found at surgery in 15 patients, and 16 lesions were correctly localized with an accuracy of $84.2 \%(16 / 19)$. $4 \mathrm{D}-\mathrm{CT}$ identified 2 potential lesions that were not adenomas, giving a PPV of $88.9 \%(16 / 18)$. No significant difference was observed between these 2 populations $(P>.56)$.

Stratification of the patients by surgery, but not by phase, also revealed no significant difference in the results obtained from the primary vs the repeated surgery data $(P>.65)$. As no differences were identified among any of the subgroups, the data were pooled and the final results were 284 parathyroid adenomas identified at surgery in 200 patients. No lesions were identified in 8 patients $(3.8 \%)$. Of the 284 lesions, 4D-CT correctly identified 233 $(82.0 \%)$. In addition to the correctly identified lesions, $4 \mathrm{D}-\mathrm{CT}$ indicated 40 potential lesions that were not found at surgery, were found to be normal glands, or were nonparathyroid structures such as exophytic thyroid nodules or lymph nodes (false-positive results) with a PPV of $85.3 \%(233 / 273)$.

Of the 200 patients in whom abnormal parathyroid tissue was found, 146 underwent unilateral neck exploration and 54 underwent bilateral neck exploration. 4D-CT correctly identified unilateral vs bilateral disease in $179(89.5 \%)$ of these 200 patients, including the mediastinal lesions. In the 146 patients who underwent unilateral surgery, 4D-CT correctly identified the side and location of the abnormal gland(s) in 126 cases (86.3\%). Considering the repeated surgery cohort separately, 4D-CT correctly discriminated between unilateral and bilateral disease in 46 (95.8\%) of the 48 cases.

Preoperative technetium Tc99m sestamibi examination results were available in 167 of 200 patients with abnormal parathyroid tissue at surgery (140 who underwent primary parathyroidectomy; 27 who underwent re-exploration surgery). Technetium Tc99m sestamibi was concordant with the surgical findings in 45 (26.9\%) of the 167 patients. In an additional 12 patients, technetium Tc99m sestamibi localized 1 lesion correctly but failed to 


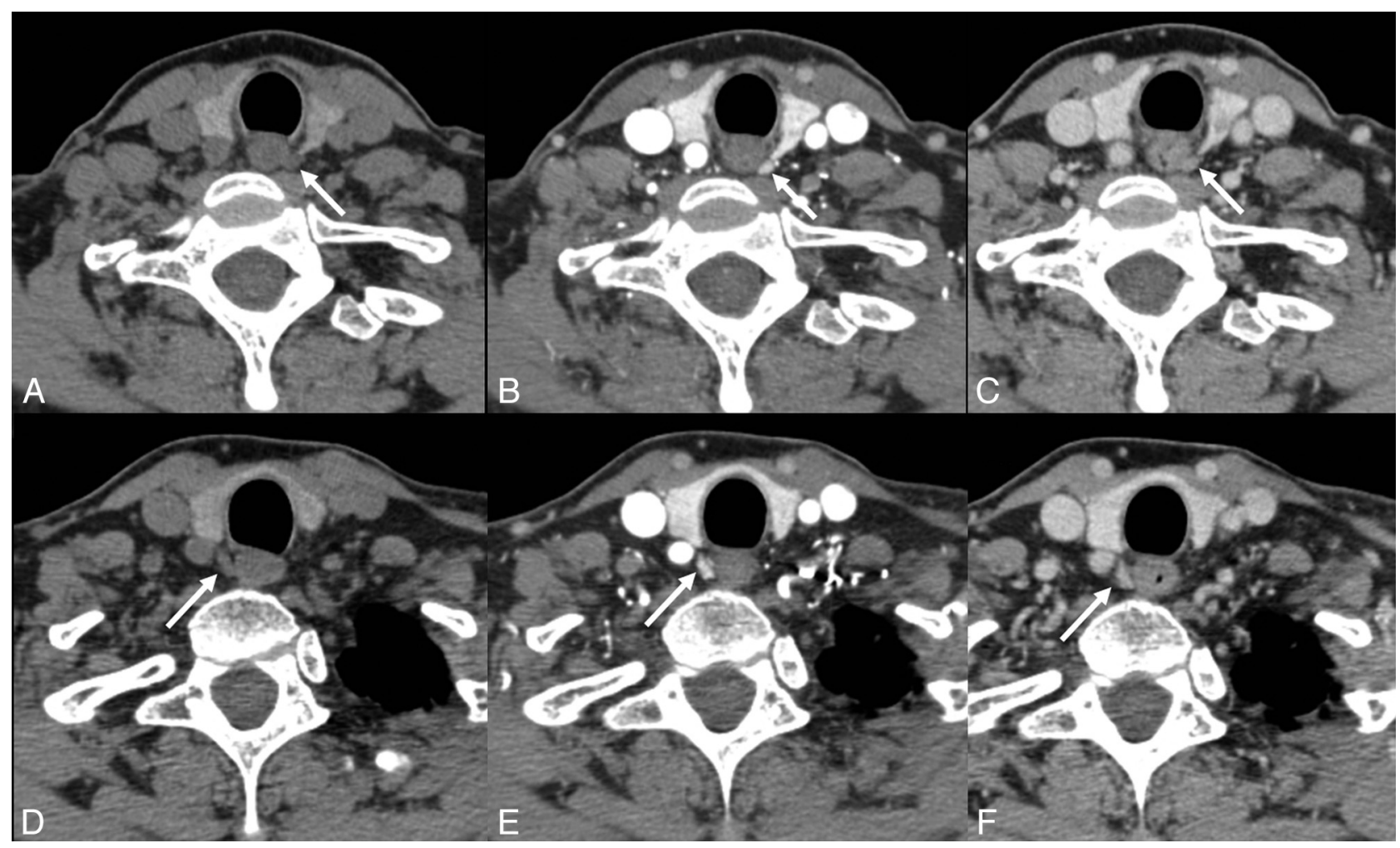

FIG 1. A 63-year-old woman with primary hyperparathyroidism. No lesions were identified on sonography or technetium Tc99m sestamibi. 4D-CT demonstrates avidly enhancing lesions in the orthotopic superior location (arrows) bilaterally with rapid washout of contrast greater than that of the adjacent thyroid gland ( $A$ and $D$ : noncontrast phase; $B$ and $E$ : initial postcontrast "arterial" phase; $C$ and $F$ : delayed postcontrast phase). This patient underwent bilateral exploration, and bilateral superior parathyroid adenomas were found at surgery.

identify additional abnormal parathyroid lesions in the same patient. Technetium Tc99m sestamibi was discordant with the surgical and pathologic findings in 110 patients (65.9\%).

Preoperative sonography examination results were available in 165 of 200 patients with abnormal parathyroid tissue at surgery (137 who underwent primary parathyroidectomy; 28 who underwent re-exploration surgery). Sonography was concordant with the surgical findings in 43 (26.1\%) of 165 patients. In an additional 18 patients, sonography localized 1 lesion correctly but failed to identify additional abnormal parathyroid lesions, or suggested additional lesions that were not present at surgery. Sonography results were discordant with the surgical and pathologic findings in 104 patients (63\%).

Abnormal parathyroid glands were identified correctly by both technetium Tc99m sestamibi and sonography in only 15 of the 208 patients. The 4D-CT and surgical findings were concordant with the technetium Tc99m sestamibi and sonography results in all 15 patients.

Fig 1 is an example of a case in which sonography and technetium Tc99m sestamibi results were negative, but 4D-CT correctly predicted bilateral disease and the locations of the abnormal parathyroid glands. Fig 2 is an example of a complicated case from the reoperative group in which $4 \mathrm{D}-\mathrm{CT}$ correctly lateralized and localized the site of disease.

\section{DISCUSSION}

Unilateral minimally invasive parathyroidectomy has become increasingly used as the favored technique for treatment of hyper- parathyroidism from any cause. ${ }^{6,7}$ Successful deployment of minimally invasive parathyroidectomy requires accurate information concerning the location of parathyroid lesions. If the preoperative localization studies indicate multigland or ectopic disease, minimally invasive parathyroidectomy may not be the correct choice, and a conventional bilateral neck exploration should be performed. In patients with no prior surgery and with only singlegland disease located in the neck, 4D-CT by use of a 4-phase protocol initially adopted at our institution has been reported to provide accuracy for localization of $93.7 \% .{ }^{12}$ In our study, a more heterogeneous group of patients has been considered, including those with multigland disease (including patients with secondary or tertiary hyperparathyroidism), those undergoing a second surgery because of persistent hyperparathyroidism, and those with parathyroid lesions in ectopic locations. Furthermore, protocols by use of 4- or 3-phase acquisition techniques have also been evaluated in these groups. Although there was variability in the localization accuracy in the subgroups, with the lowest accuracy seen in the 4-phase, primary surgery cohort (77.9\%) and the highest accuracy in the 3-phase, primary surgery cohort (87.1\%), statistical analysis indicated that all of the subgroups were equivalent $(P>.56)$, with a pooled accuracy of $82 \%$.

Arguably the most challenging patients are those with multigland and/or recurrent disease who are undergoing a second surgery. In this particular subgroup, localization accuracy was $84 \%$ in our study, whereas previous studies by other groups recorded accuracies of $88 \%$ and $73 \%{ }^{16,14}$ in similar patient cohorts. Vari- 


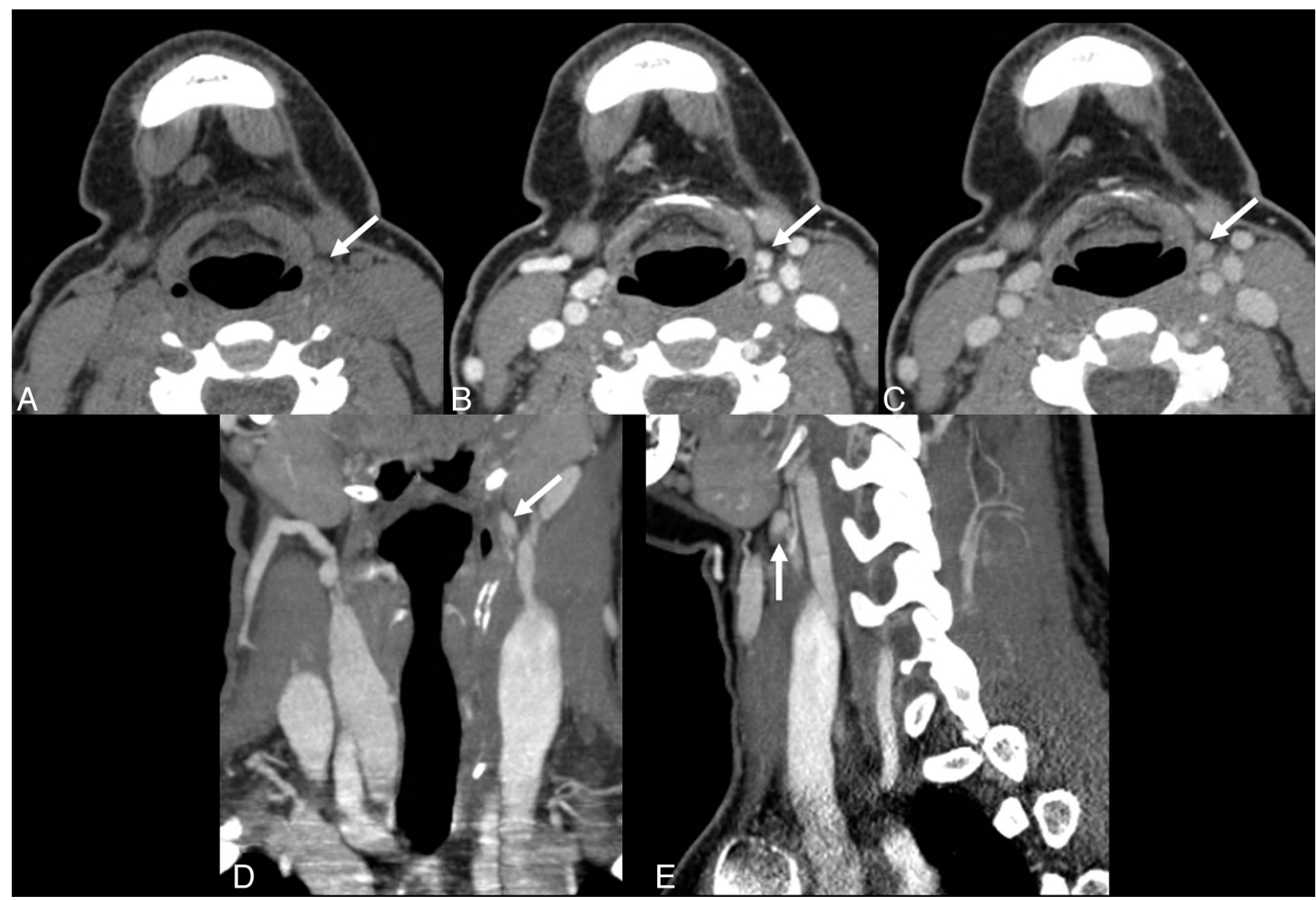

FIG 2. A 26-year-old woman with persistent primary hyperparathyroidism after undergoing a 7-hour neck exploratory procedure including the upper mediastinum and a left hemithyroidectomy, as the left inferior gland could not be found. 4D-CT demonstrates a small lesion high in the left neck at the level of the hyoid bone (arrows). Perfusion characteristics are suggestive of a parathyroid adenoma, with a lesion lower in attenuation than the thyroid gland on the initial noncontrast phase $(A)$, and rapid uptake of contrast $(B)$ and rapid washout of contrast $(C)$, greater than that of the thyroid gland (not shown). ( $D$ : coronal reformatted image in the "arterial" phase; $E$ : sagittal reformatted image in the "arterial" phase). At surgery, a parathyroid adenoma was found in the left carotid sheath, at the apex of ectopic thymic tissue, consistent with an undescended left inferior parathyroid gland.

ability in these results likely reflects the small numbers of patients studied: 48 in our group, 45 in the study by Mortenson et al, ${ }^{16}$ and 21 in the study by Lubitz et al. ${ }^{14}$ Although overall localization accuracy was $82 \%$, discrimination between unilateral and bilateral disease in this cohort was successful in 46 (95.8\%) of 48 patients. This improvement is important because surgery in the reoperative neck is often more difficult, and patients are at increased risk for significant morbidity. ${ }^{16}$ Improved preoperative localization and lateralization may prevent unnecessary dissection and complications. ${ }^{16,19}$

In our patient population, neither sonography nor technetium Tc99m sestamibi scanning provided adequate localization. The success rate for localization by technetium Tc99m sestamibi and/or sonography was approximately $27 \%$ in our 200 unselected patients. This rate was markedly lower than that recorded in the literature. ${ }^{8}$ The low accuracies and rates of concordance of these primary localization methods in our cohort likely reflect the referral pattern of the endocrine surgeons in our practice. Deployment of $4 \mathrm{D}-\mathrm{CT}$ is biased toward complex or difficult cases, ie, when sonography and/or technetium Tc99m sestamibi results are negative, or if the results of those 2 studies are discordant. Thus, our cohort consists of unselected, potentially complex patient cases, and this trend is reflected in lower identification rates com- pared with the results from cohorts of relatively uncomplicated patient cases. ${ }^{12,13}$

Many different protocols have been advocated in the literature for $4 \mathrm{D}$-CT. These are typically 2 -phase scans with imaging acquired only after contrast ${ }^{20,21}$ or imaging acquired both before and after contrast. ${ }^{11}$ Assessment of such protocols demonstrates significantly lower rates of accuracy compared with our current 3-phase protocol (Hunter GJ, Ginat DT, Kelly HR, et al; unpublished data, 2013).

The change from a 4 - to a 3 -phase protocol was made to reduce the effective dose of a $4 \mathrm{D}-\mathrm{CT}$ study from approximately $28 \mathrm{mSv}$ to $21 \mathrm{mSv} .{ }^{12}$ We believe the benefit of accurate localization represents a favorable risk-benefit ratio to patients needing surgery for hyperparathyroidism. The current 3-phase protocol balances the need for sufficient data against unnecessary phases. As our results demonstrate, the move from 4 to 3 phases has not decreased accuracy in localization but has decreased the effective dose. Further reduction to a 2- or singlephase study is likely to be counterproductive, as the decrease in effective dose (an indeterminate delayed risk) may result in a disproportionally greater decrease in accuracy of localization (an immediate benefit).

There were several limitations in this study. First, this study 
was retrospective and could not prospectively address the accuracy of 4D-CT in all patients with hyperparathyroidism. As multigland disease and ectopic glands are more likely to be missed by the traditional initial imaging studies of sonography and technetium Tc99m sestamibi, our patient population was also likely skewed toward more complex or difficult cases by the referral bias mentioned above. Furthermore, the accuracies reported in this study reflected the results of $4 \mathrm{D}-\mathrm{CT}$, as interpreted by a single neuroradiologist with 7 years of experience with this technique and evaluation of more than 900 cases. Therefore, our study was not able to assess interobserver variability. Interpretation of the images is time consuming and, in our cohort, may be nontrivial; if adequate time is not devoted to interpretation, accuracy rates will be compromised. Finally, the overall accuracy of 4D-CT is likely to vary by institution, CT scanner, experience and interest of the interpreter, and CT protocol technique used to generate the images.

\section{CONCLUSIONS}

4D-CT is an accurate technique for preoperative localization of parathyroid lesions in patients with hyperparathyroidism regardless of cause or prior parathyroid surgical history, and can be used to stratify patients to unilateral minimally invasive parathyroidectomy vs bilateral neck exploration.

\section{ACKNOWLEDGMENTS}

The authors thank Dr. Elkan Halpern for assistance in providing statistical advice during manuscript revision.

\section{REFERENCES}

1. The American Association of Clinical Endocrinologists and the American Association of Endocrine Surgeons position statement on the diagnosis and management of primary hyperparathyroidism. Endocr Pract 2005; 11:49-54

2. Fraser WD. Hyperparathyroidism. Lancet 2009;374:145-58

3. Tominaga Y, Tanaka Y, Sato K, et al. Histopathology, pathophysiology, and indications for surgical treatment of renal hyperparathyroidism. Semin Surg Oncol 1997;13:78-86

4. Greene AB, Butler RS, McIntyre S, et al. National trends in parathyroid surgery from 1998 to 2008: a decade of change. J Am Coll Surg 2009;209:332-43

5. Udelsman R, Lin Z, Donovan P. The superiority of minimally invasive parathyroidectomy based on 1650 consecutive patients with primary hyperparathyroidism. Ann Surg 2011;253:585-91
6. Kunstman JW, Udelsman R. Superiority of minimally invasive parathyroidectomy. Adv Surg 2012;46:171-89

7. Fraker DL, Harsono H, Lewis R. Minimally invasive parathyroidectomy: benefits and requirements of localization, diagnosis, and intraoperative PTH monitoring. Long-term results. World J Surg 2009;33:2256-65

8. Johnson NA, Carty SE, Tublin ME. Parathyroid imaging. Radiol Clin North Am 2011;49:489-509

9. Rodgers SE, Hunter GJ, Hamberg LM, et al. Improved preoperative planning for directed parathyroidectomy with 4-dimensional computed tomography. Surgery 2006;140:932-40

10. Beland MD, Mayo-Smith WW, Grand DJ, et al. Dynamic MDCT for localization of occult parathyroid adenomas in 26 patients with primary hyperparathyroidism. AJR Am J Roentgenol 2011;1961:61-65

11. Kutler DI, Moquete R, Kazam E, et al. Parathyroid localization with modified 4D-computed tomography and ultrasonography for patients with primary hyperparathyroidism. Laryngoscope 2011;121:1219-24

12. Hunter GJ, Schellingerhout D, Vu TH, et al. Accuracy of fourdimensional CT for the localization of abnormal parathyroid glands in patients with primary hyperparathyroidism. Radiology 2012;264:789-95

13. Cheung K, Wang TS, Farrokhyar F, et al. A meta-analysis of preoperative localization techniques for patients with primary hyperparathyroidism. Ann Surg Oncol 2012;19:577-83

14. Lubitz CC, Hunter GJ, Hamberg LM, et al. Accuracy of 4-dimensional computed tomography in poorly localized patients with primary hyperparathyroidism. Surgery 2010;148:1129-37

15. Eichhorn-Wharry LI, Carlin AM, Talpos GB. Mild hypercalcemia: an indication to select 4-dimensional computed tomography scan for preoperative localization of parathyroid adenomas. Am J Surg 2011;201:334-38

16. Mortenson MM, Evans DB, Lee JE, et al. Parathyroid exploration in the reoperative neck: improved preoperative localization with 4Dcomputed tomography. J Am Coll Surg 2008;206:888-95

17. Chazen JL, Gupta A, Dunning A, et al. Diagnostic accuracy of 4D-CT for parathyroid adenomas and hyperplasia. AJNR Am J Neuroradiol 2012;33:429-33

18. Starker LF, Mahajan A, Bjorklund $\mathrm{P}$, et al. $4 \mathrm{D}$ parathyroid $\mathrm{CT}$ as the initial localization study for patients with de novo primary hyperparathyroidism. Ann Surg Oncol 2011;18:1723-28

19. Lew JI, Solorzano CC. Surgical management of primary hyperparathyroidism: state of the art. Surg Clin North Am 2009;89:1205-25

20. Gafton AR, Glastonbury CM, Eastwood JD, et al. Parathyroid lesions: characterization with dual-phase arterial and venous enhanced CT of the neck. AJNR Am J Neuroradiol 2012;33:949-52

21. Welling RD, Olson JA Jr, Kranz PG, et al. Bilateral retropharyngeal parathyroid hyperplasia detected with $4 \mathrm{D}$ multidetector row $\mathrm{CT}$. AJNR Am J Neuroradiol 2011;32:E80-82 